\title{
Psychological therapy reduced depression earlier (4 months) but at 1 year was not better than usual general practitioner care
}

\author{
Ward E, King M, Lloyd M, et al. Randomised controlled trial of non-directive counselling, cognitive-behaviour therapy, \\ and usual general practitioner care for patients with depression. I: Clinical effectiveness. BMJ 2000 Dec 2;321:1383-8. \\ QUESTION: In patients with depression, is psychological therapy (non-directive \\ counselling or cognitive behavioural therapy [CBT]) more effective than usual general \\ practitioner (GP) care?
}

\section{Design}

Randomised (allocation concealed*), unblinded,* controlled trial with 1 year follow up. This abstract reports the results of the randomised 3 way comparison only (197 of 464 patients).

\section{Setting}

13 general practices in northern London and 11 practices in greater Manchester, UK.

\section{Patients}

197 patients who were $\geqslant 18$ years of age (mean age $37 \mathrm{y}$, $77 \%$ women) and were depressed or depressed and anxious (score $\geqslant 14$ on Beck Depression Inventory [BDI]). Exclusion criteria were serious suicidal intent, psychological treatment in previous 6 months, use of antidepressants, restricted mobility, organic brain syndromes, or inability to complete questionnaires. Follow up was $91 \%$ at 4 months and $84 \%$ at 1 year.

\section{Intervention}

Patients were allocated to non-directive counselling $(n=67)$, CBT $(n=63)$, or usual GP care $(n=67)$. Psychological treatment was given in the GP's office according to a manual developed for each group. Non-directive counselling was based on the work of Rogers $\dagger$, and CBT involved problem formulation and staged intervention. Patients were initially offered 6 sessions (maximum of 12). Patients in the CBT group had a mean of 5.0 (SD 3.5) sessions, and 9 patients (14\%) did not attend any sessions; in the non-directive counselling group, patients had a mean of 6.4 (SD 4.2) sessions, and 7 patients (11\%) did not attend any sessions.

\section{Main outcome measure}

Depression (score on the BDI).

\section{Main results}

Analysis was by intention to treat. At 4 months, patients in the psychological treatment groups had greater reductions in BDI scores than those in the usual GP care group \{mean score decreases $4.5,95 \%$ CI 0.7 to 8.3 for CBT; and 5.7, CI 2.1 to 9.3 for non-directive counselling\}; at 12 months, groups no longer differed \{mean score differences $-0.9, \mathrm{CI}-4.2$ to 2.2 for CBT $v$ usual GP care; and 0.9, CI -2.4 to 4.2 for non-directive counseling $v$ usual GP care $) \ddagger$ (table).

\section{Conclusion}

In patients with depression, psychological treatment was better than usual general practitioner care for reducing depression at 4 months, but the difference no longer existed at 1 year.

*See glossary.

$\dagger$ Rogers CR. On becoming a person: a therapist's view of psychotherapy. Boston: Houghton Mifflin; 1961.

$\ddagger$ Mean differences and CIs calculated from data in article.

Non-directive counseling or cognitive behavioral therapy (CBT) v usual general practitioner $(G P)$ care for depression§

\begin{tabular}{lccl} 
& \multicolumn{3}{l}{ Mean BDI score (SD) } \\
\cline { 2 - 4 } Follow-up & CBT & Counseling & GP care \\
Baseline & $27.6(8.4)$ & $25.4(8.6)$ & $26.5(8.9)$ \\
\hline 4 months & $12.7(9.5)$ & $11.5(7.7)$ & $17.2(11.9)$ \\
\hline 12 months & $9.3(8.8)$ & $11.1(9.3)$ & $10.2(8.5)$ \\
\hline
\end{tabular}

$\S \mathrm{BDI}=$ Beck Depression Inventory.

2 Friedli K, King MB, Lloyd M, et al. Randomised controlled assessment of non-directive psychotherapy versus routine general-practitioner care. Lancet 1997;350:1662-5. 